\title{
DÉMOCRATIE, HOSPITALITÉ ET VIOLENCE. INTERVIEW AVEC MARC CRÉPON
}

\section{DEMOCRACY, HOSPITALITY AND VIOLENCE. INTERVIEW WITH MARC CRÉPON}

\author{
Verónica GonZÁlez et Javier AgÜERo ÁGUILA*
}

Marc Crépon est directeur de recherche au Centre national de la recherche scientifique (CNRS) et directeur du département de philosophie à l'École normale supérieure (ENS), Paris, France. Il a publié entre autres : La culture de la peur, I. Démocratie, identité, sécurité (Galilée, 2008); La culture de la peur, II. La guerre des civilisations (Galilée, 2010) ; Le consentement meurtrier (Cerf, 2012); La vocation de l'écriture (Odile Jacob, 2014); La gauche c'est quand? (Des Equateurs, 2015).

VERÓNICA GONZÁLEZ : L'interview s'organise autour de trois axes qui nous semblent contenir les problématiques politiques actuelles présentes dans votre travail. Le premier axe concerne la démocratie et la culture de la peur, le deuxième porte sur la démocratie et l'hospitalité et le troisième a trait à la politique, à la violence et au langage.

\footnotetext{
* Verónica González est doctorante en philosophie, esthétique et théorie de l'art de l'Université du Chili et boursière CONICYT (Commission Nationale de la Recherche Scientifique et Technologique du Gouvernement Chilien). Javier Agüero Águila est docteur en philosophie de l'Université Paris 8, membre du laboratoire d'études sur les logiques contemporaines de la philosophie (LLCP) et boursier CONICYT.
} 
Vous avez écrit sur la peur, sur une sorte de culture de la peur qui aurait trouvé aujourd'hui un terrain fertile pour germer et se propager. Dans cette perspective, vous remarquez que les États démocratiques transforment cette culture et qu'elle devient un principe de gestion et d'administration du pouvoir. La démocratie aurait alors tendance à se confondre avec d'autres régimes dont elle voudrait se distinguer et s'opposer (par exemple, les totalitarismes). Quelle devrait être selon vous une démocratie dont le principe est de neutraliser et de s'opposer à cette culture de la peur?

MARC CRÉPON : D'abord, la première chose que je voudrais dire, c'est qu'il ne s'agit pas de diaboliser la peur, dès lors que la peur est légitime et qu'il y a objectivement des raisons d'avoir peur. On peut distinguer en effet divers domaines de l'existence, qui sont favorables au développement et même à la progression de cette émotion. Il est légitime d'avoir peur pour sa santé, de s'inquiéter pour l'état de la planète, de l'environnement, etc. Et que dire de la peur de perdre son emploi ! De la peur de se retrouver au chômage et de celle d'une dégringolade sociale. Toutes ces peurs-là qui traversent les sociétés sont des peurs légitimes, sans compter les peurs réelles liées à la situation internationale, parce qu'on vit dans un monde objectivement menaçant. Il ne faudrait pas, par conséquent, se faire une image exclusivement péjorative de la peur.

Maintenant, une fois qu'on a dit cela, il faut analyser la façon dont les États démocratiques gèrent la peur du citoyen, les émotions du citoyen. Traditionnellement on peut dire que les régimes non-démocratiques se sont toujours appuyés sur la peur. Ils l'ont toujours entretenue, ils l'ont toujours développée, ils ont fait de la peur du citoyen un des instruments de la conservation du pouvoir. Et à l'inverse, on pouvait penser que les régimes démocratiques se distinguaient des régimes non-démocratiques en ceci qu'ils n'avaient pas besoin de cette peur pour exercer le pouvoir. Ce qui crée aujourd'hui une confusion c'est que les gouvernements démocratiques ont compris - c'est plus visible maintenant - qu'ils pouvaient instrumentaliser les émotions des citoyens pour consolider leur légitimité. Or, cette instrumentalisation est à géométrie variable, car elle consiste pour les gouvernements démocratiques, comme on l'a pu observer ces dernières années, à fabriquer ce que le sociologue Zygmunt Bauman appelle des " cibles de substitution ». Cela revient pour le gouvernement à choisir, entre les différentes peurs des citoyens, celles pour lesquelles on va désigner une cible et qu'il va assurer, déclarer pouvoir prendre en charge et celles qu'il déclarera ne 
pas être complètement de son ressort. C'est ainsi que les gouvernements pourront admettre que les citoyens aient peur de perdre leur travail, qu' ils redoutent les effets désastreux de la mondialisation sur l'emploi, reconnaître le sentiment d'insécurité qui en résulte, et avouer en même temps qu'ils ne peuvent pas faire grand-chose contre cette insécurité, dès lors qu'ils ne maitrisent pas les effets de la mondialisation. C'est ainsi que les sociétés démocratiques voient se développer un chômage endémique, une pauvreté croissante, sans que la peur qui en résulte chez les citoyens soit ce dont les gouvernements se préoccupent prioritairement. D'où les cibles de substitution : les étrangers, les immigrants, les délinquants. Le phénomène fut particulièrement sensible lors du dernier quinquennat, lorsque le précédent président de la République en France, Nicolas Sarkozy, s'attachait, tous les six mois, à offrir à l'opinion publique une nouvelle cible de substitution.

Donc ce que j'appelle la culture de la peur consiste en deux choses. Cela consiste d'une part, dans la fabrication de ces cibles de substitution et, d'autre part, dans le refus face aux peurs des citoyens de prendre le parti d'un discours rationnel, de surfer donc en permanence sur les émotions des citoyens au lieu de tenter de les corriger par un discours de cet ordre.

JAVIER AGÜERO : Vous venez de parler de la figure de l'immigrant, de l'étranger, qui est également au centre de votre travail. En considérant la problématique que vous venez d'approfondir à propos de la démocratie et de la culture de la peur, il nous semble qu'il n'y ait pas un rapport essentiel entre démocratie et hospitalité, mais plutôt que l'hospitalité est une exigence - qui comme toute exigence est externe - à laquelle les démocraties occidentales doivent donner une réponse. Est-ce que vous pensez que la démocratie et l'hospitalité sont deux instances consubstantielles ou bien qu'elles sont forcées à se rencontrer?

M. C. : Pour moi elles sont vraiment consubstantielles, parce qu'au fond ce qui devrait distinguer la démocratie de tous les autres régimes politiques c'est que l'appartenance au corps des citoyens n'est conditionnée par aucune autre forme d'appartenance, c'est-à-dire que pour être citoyen d'une démocratie vous ne devriez pas y avoir à faire état d'une autre forme d'appartenance quelle qu'elle soit, qu'elle soit d'ordre religieux, ethnique, culturel, linguistique, etc. Je pense qu'une démocratie c'est un système politique dans lequel l'appartenance 
est non conditionnelle. Alors que toutes les formes de restriction de l'hospitalité consistent à mettre des conditions. Donc, il y a un lien consubstantiel entre la démocratie et l'hospitalité et je pense qu'aujourd'hui - pour dire les choses dans un autre sens - l'une des grandes forces hostiles qui menace les démocraties, notamment les démocraties européennes, c'est la xénophobie qui se développe et qui consiste à stigmatiser l'étranger, le réfugié, l'immigré. Elle entraîne le développement de forces politiques effectivement réactionnaires, racistes, dont les valeurs, les principes, sont, à mon sens, complètement hostiles et contraires à ceux de la démocratie.

Je pense, en outre, que les valeurs de la république, qui sont la liberté, l'égalité, la fraternité, définissent aussi le socle de principes sur lesquels devraient reposer les démocraties européennes ; et que lorsqu'on commence à vouloir multiplier les conditions de l'hospitalité (quand on accepte que s'installe progressivement dans l'opinion publique une façon de parler des étrangers, des immigrés, des réfugiés comme si c'était un problème et qu'on les stigmatise), on porte atteinte à ces valeurs fondamentales sur lesquelles repose notre attachement à la démocratie et à la république. Aujourd'hui cette question de l'hospitalité est au cœur de ce qui fragilise les démocraties européennes, ainsi que la construction de l'Europe, comme on l'a vu cet été avec la crise des réfugiés. Parce qu'elle se traduit par la montée en puissance de forces politiques dont toute l'histoire, tout l'héritage, sont contraires non seulement aux valeurs de la république, mais également à celles de la démocratie.

La démocratie et l'hospitalité sont consubstantielles, car on ne peut pas conditionner l'appartenance au corps des citoyens. Dans l'essence de la démocratie il y a quelque chose qui suppose l'hospitalité, qui suppose l'accueil des étrangers, l'ouverture à l'autre. Voilà le premier point. Ensuite, elles sont consubstantielles, parce qu'aujourd'hui c'est un combat politique, et que ceux qui sont hostiles à cette ouverture sont les mêmes qui historiquement se sont opposés, à un moment ou à un autre de notre histoire, à la démocratie.

J. A. : Peut-on penser que tous ceux qui refusent d'accueillir l'étranger sont aussi antidémocrates? 
M. C. : Je pense qu'il y a une incompatibilité fondamentale entre la xénophobie (conçue comme la peur de l'étranger) et les valeurs dont peuvent se réclamer les démocraties. Voilà une position qu'il faut tenir avec la plus grande fermeté.

J. A. : Dans votre dernier débat avec Alain Finkielkraut, intitulé Comment peut-on être étranger ?', qui concerne le phénomène d'intégration des immigrants, vous avez soutenu quelque chose qui nous a semblé pour le moins inquiétant. Vous avez dit, en essayant de répondre aux arguments de Finkielkraut qui nous semblent la plupart du temps très localisés, que Derrida n'était pas l'apôtre de l'hospitalité inconditionnelle. C'est intéressant parce qu'il est vrai que Derrida pense l'inconditionnel comme une sorte d'exigence éthique du conditionnel, ou bien comme quelque chose qui devrait faire pression sur le conditionnel, de telle manière que la dimension politique et les lois elles-mêmes soient créées en considérant cet espace de l'incalculabilité et de l'hyperbolique. Néanmoins, il y a beaucoup de passages chez Derrida où il explique que l'hospitalité inconditionnelle doit rester pure (Le monolinguisme de l'autre, entre autres), absolue et ne peut être ni politique ni réglée par la loi et, en fin de compte, que l'hospitalité inconditionnelle ne peut pas être publique, tout sauf publique.

Est-ce que vous croyez finalement qu'au-delà du conditionnel, Derrida n'est pas un apôtre de l'inconditionnel? Dans le sens même d'apôtre, c'est-à-dire celui qui marche toujours derrière quelque chose ou quelqu'un, dans ce cas de l'inconditionnel ou de l'impossible?

M. C. : D'abord il faut savoir que cette idée très forte de l'hospitalité inconditionnelle se prête à beaucoup de caricatures, très souvent opposées, utilisées contre Derrida en disant qu'il est l'apôtre d'une hospitalité inconditionnelle qui est impraticable. Dit-on : "Comment voulez-vous qu'un État ne mette pas des limites à l'hospitalité ! ", " On ne peut pas accueillir tout le monde !», etc. Donc, dire que Derrida n'est pas un apôtre de l'hospitalité inconditionnelle, c'est s'en prendre à ces résumés caricaturaux et péjoratifs de sa pensée qui est infiniment plus complexe.

${ }^{1}$ Débat publié dans Philosophie magazine n86, Paris, février 2015, pp. 60-65. 
Par ailleurs, le mot d'apôtre convient très mal à Derrida, car il n'aurait pas voulu lui-même se reconnaître comme l'apôtre de quoi que ce soit, y compris même de la déconstruction.

Il faut dire que l'opposition de l'hospitalité conditionnelle et de l'hospitalité inconditionnelle, les tensions qui existent entre les deux recoupent très précisément la tension qui existe entre le droit et la justice, qui est une tension aporétique, irréconciliable. Cette tension irréconciliable signifie qu'au fond le droit et la justice appartiennent à des ordres hétérogènes - comme l'hospitalité conditionnelle et l'hospitalité inconditionnelle -, mais cela ne veut pas dire qu'il n'y a pas de relation entre les deux. Cela rappelle que, quelles que soient les conditions que vous imposez à l'hospitalité, celles-ci ne sont pas de l'ordre de la justice, elles ne peuvent pas se donner pour la justice. Voilà l'essentiel : la justice ne peut jamais être du côté du conditionnel. Ces conditions supposent toujours une transaction avec un principe hétérogène, radicalement hétérogène, qui est l'hospitalité inconditionnelle et qui est de l'ordre de la justice. Ça fait une énorme différence. La différence n'est pas entre l'hospitalité conditionnelle et l'hospitalité inconditionnelle, aujourd'hui la différence est entre une hospitalité conditionnelle qui n'est en tension avec rien d'autre qu'elle-même - et, dans ce cas, on peut multiplier à l'infini les conditions, on peut s'engager dans une politique qui va rendre de plus en plus difficile, de plus en plus impraticable, l'accueil de l'étranger - et une politique qui sait que les conditions qu'elle impose à l'hospitalité sont juridiques, qu'elles sont peut-être une nécessité, mais qu'elles ne peuvent jamais se réclamer de la justice.

Voilà pourquoi Derrida n'est pas l'apôtre de l'hospitalité inconditionnelle qui ne voudrait rien entendre, qui ne voudrait rien savoir des conditions de l'hospitalité conditionnelle. Derrida est quelqu'un qui rappelle aux conditions de l'hospitalité que pratiquent les Etats qu'elles ne peuvent pas se réclamer de la justice, parce que la seule justice qui tienne serait celle d'une hospitalité inconditionnelle. Dès qu'il y a des conditions, on n'est plus dans la justice, mais dans une transaction impossible avec son principe.

Ce n'est pas la première fois que je débattais avec Alain Finkielkraut sur cette question. J'avais été invité à la fameuse émission du samedi matin pour débattre sur l'Europe avec un philosophe de droite très radical, très extrémiste qui, connaissant le lien qui m'attache à la pensée de Derrida, m'accusait d'une façon très caricaturale, en me demandant comment je pouvais m'intéresser à cet apôtre de l'hospitalité inconditionnelle, irréaliste, qui voudrait que les États 
renoncent à toute règle nette, toute norme pour réguler l'accueil des étrangers. Et c'est alors que j'ai commencé à expliquer que sa position était plus compliquée que cela et qu'il n'y avait pas d'hospitalité inconditionnelle qui soit pensable indépendamment de sa tension avec des règles de l'hospitalité conditionnelle propres à chaque État.

J. A. : Lapôtre est celui qui marche derrière quelque chose. Dire que Derrida n'est pas l'apôtre de l'hospitalité inconditionnelle peut conduire à croire qu'il ne marche pas derrière l'hospitalité inconditionnelle.

M. C. : L'apôtre n'est pas seulement celui-là. L'apôtre c'est celui qui porte le message de l'Évangelie, c'est l'annonciateur d'une bonne nouvelle - et il n'y a pas de bonne nouvelle. Je ne pense pas qu'un philosophe puisse être apôtre de quoi que ce soit - même s'il y a certains philosophes qui se croient des apôtres. Je comprends que vous vous posiez la question. Moi aussi, je me la suis posée, parce que si Derrida n'est pas un annonciateur de bonnes nouvelles, il joue tout le temps avec deux catégories très proches, difficilement indissociables de l'idée d'annonce : celle de la promesse et celle de la messianicité sans messianisme. Toute sa pensée d'une démocratie à-venir annonce un évènement à-venir, un évènement qui provient du futur, dont la structure relève de cette logique de la messianicité sans messianisme. Sans doute tout ceci n'est-il pas sans lien (possible) avec la figure de l'apôtre. Mais toute la question porte alors sur le statut de celui qui annonce cette démocratie à-venir : Est-ce qu'il la pense seulement? Ou est-ce qu'il l'annonce comme prophète, est-ce qu'il la défend comme apôtre?

Ce qui est vrai, c'est que l'idée d'une démocratie à-venir est absolument indissociable du rapport entre l'hospitalité conditionnelle et l'hospitalité inconditionnelle. La démocratie à-venir est une démocratie qui porte une idée de la justice qui intègre l'idée d'une hospitalité inconditionnelle.

V. G. : Dans votre livre La vocation de l'écriture, qui a été publié en 2014, vous soutenez qu'il y a une violence inscrite au cœur de l'expérience du langage, c'est-à-dire une sorte de violence constitutive du langage qu'on apprend dans sa 
famille, à l'école, au sein du système éducatif et du milieu social. Vous faites la distinction entre diverses formes de cette violence. La plus radicale serait celle qui provient de toutes ces caractérisations indues qui réduisent l'autre à n'importe quelle forme d'appartenance ou d'identité collective ou communautaire, parce qu'elle efface et annule sa singularité. Vous faites référence aux phénomènes du racisme, à l'antisémitisme, mais il semble que vous vouliez, de façon plus large, désigner une violence inscrite dans le langage comme instance générale. Cependant, vous soutenez que c'est le langage lui-même qui nous donne la possibilité sinon de dépasser, au moins de contredire cette violence. Vous renvoyez alors à une autre expérience du langage, à savoir : l'événement possible d'une contreparole qui répond à la violence par l'invention d'une singularité. Vous retrouvez cette possibilité dans la littérature et dans la philosophie, dans la mesure où faisant l'épreuve de la violence, elles affirment ce que vous nommez « une invention 'idiomatique' de la singularité».

Nous aimerions que vous développiez ce dernier point, mais avant tout, considérant le séminaire que vous donnez à l'ENS intitulé Contrer la violence, nous souhaiterions vous poser la question suivante. Peut-on imaginer un discours politique qui refuserait la violence inhérente à son langage, alors que cette même violence est constitutive de la politique ? En effet, vous-même écrivez : "Toute politique suppose l'appartenance, circonscrit l'attachement et impose l'exclusion, sous une forme déterminée qui, nécessairement, porte la violence $»^{2}$.

M. C. : Je vais tenter de répondre d'abord à la question du lien consubstantiel entre la violence et le langage. Ce que j'ai voulu rappeler dans la très longue introduction de La vocation de l'écriture, c'est la façon dont il y a dans la genèse de toute subjectivité un lien entre l'expérience de la violence et l'expérience du langage. D'abord parce que l'apprentissage du langage sous toutes ses formes ne se fait pas sans violence, sans contraintes, il ne se produit pas sans l'assimilation d'un certain nombre de lois qui régissent sa pratique et son usage, qui sont en elles-mêmes violentes. Lorsque nous parlons nous nous faisons violence; il y a ce que nous voulons dire, ce que nous ne voulons pas dire, ce que nous osons dire, ce que nous n'osons pas dire, ce que nous n'arrivons pas à dire - mille et une expériences donc qui attestent ce lien ténu, subtil entre l'expérience du langage et

${ }^{2}$ Marc Crépon, «La peur des étrangers» dans : La culture de la peur, I. Démocratie, identité, sécurité, Paris, Galilée, 2008, p. 117. 
l'expérience de la violence. On grandit avec, on vit avec, et l'on peut même dire que l'un des principaux défis de l'existence est d'avoir un rapport au langage qui l'affranchisse de la violence. Voilà sans doute la première, la plus générale et la plus forte définition de l'éthique. L'éthique c'est l'invention d'une contre-parole dans la relation à l'autre qui la libère de la violence.

Quelle est là-dedans la place de la politique ? A quoi tient le lien consubstantiel de la politique et de la violence? A ceci d'abord que toute politique est exclusive dès lors qu'elle est circonscrite dans les limites d'un État donné. Une politique déterminée produit donc toujours de l'exclusion. Ensuite, le langage politique, la langue que parle la politique elle-même est une langue qui, de façon plus ou moins visible et sensible, est habitée par la violence. Même les régimes démocratiques, qui semblent être les plus éloignés de la violence, n'échappent pas à ce rapport constitutif entre violence et politique. Face à la politique la nécessité s'impose donc d'une contre-parole : l'une des tâches indispensables de la philosophie, c'est la critique. C'est pourquoi les philosophes ne doivent pas s'intéresser uniquement à ce que disent et écrivent des philosophes, mais aussi aux langues multiples de la politique pour repérer en elles la violence qui n'est pas toujours explicitement visible. Je pense que c'est vraiment cela qui est une des forces de la déconstruction dans le sens derridien, c'est traquer dans les usages du langage $-\mathrm{y}$ compris dans les usages politiques - cette violence constitutive.

V. G. : À votre avis, cette contre-parole doit rester en dehors de l'espace politique?

M. C. : Non, pas nécessairement.

V. G. : Une contre-parole en politique est-elle possible?

M. C. : Oui, quand des politiques héritent de la violence, il leur revient d'imaginer une langue publique commune qui sorte la politique de cette violence. 
Les grandes scènes de réconciliation et de pardon, par exemple, sont des situations qui essayent d'imaginer une langue commune qui sorte de la spirale de la violence dans laquelle le discours est plongé. Dans mon séminaire, si vous êtes là les mois qui viennent, on va traiter Martin Luther King et Nelson Mandela, la lutte pour les droits civiques et les luttes contre l'apartheid. J'essaierai d'y montrer les différents éléments de langage que proposent Nelson Mandela et Desmond Tutu pour sortir la politique de son enfermement dans la violence. Comment sortir de la violence, voilà ce qui m'intéresse. Comment le discours politique peut-il (et doit-il) se laisser questionner, déranger, ébranler par des principes hétérogènes, comme ceux de l'éthique? Nous en avons un exemple avec la tension, dont nous venons de parler entre hospitalité conditionnelle et hospitalité inconditionnelle. L'opposition que nous avons décrite, une autre manière de la penser est de dire qu'elle distingue une politique de l'hospitalité qui ne veut pas se laisser travailler par aucun principe éthique et une politique qui, au contraire, accueille en elle ses injonctions.

Parlons de choses très concrètes, à savoir de cette crise majeure des réfugiés en Europe! Il y a des associations qui travaillent pour accueillir des réfugiés, pour les prendre en charge, pour les aider à remplir les formalités nécessaires, des associations qui luttent pour imposer à des situations terribles des règles qui ne sont pas celles de la politique, et qui sont inspirées par des principes éthiques forts (solidarité, fraternité, générosité, respect de la dignité). Et bien ce qui les caractérise, c'est leur ancrage éthique. Et cela fait une différence énorme de considérer que tous ces principes-là doivent être pris en considération ou de penser l'inverse. Il y a une différence entre penser que l'accueil des réfugiés doit obéir à un impératif éthique qui inspire l'action et le discours, et considérer à l'inverse que c'est un problème technique et politique qui doit être réglé en dehors de toute considération éthique (de fraternité, de solidarité, etc.).

V. G. : Je voudrais insister sur la question de la contre-parole dans la littérature et dans la philosophie qui sont des lieux extérieurs à la politique. Peut-on penser cette contre-parole comme une condition de possibilité de la politique?

M. C. : En tout cas d'une politique démocratique. Il faut tenir tous les fils ensemble. Si on parle de la démocratie à-venir, c'est une autre démocratie, portée 
par une idée de la justice. Elle suppose à chaque fois ce que Derrida appelle une invention idiomatique. On ne peut pas séparer le motif de la démocratie à-venir de l'hospitalité inconditionnelle, de la traduction, de l'idiome, tout ça se rejoint, se coordonne. Je suis convaincu qu'une autre démocratie suppose l'invention d'une contre-parole. Aujourd'hui c'est tellement difficile de se faire entendre sur la question de l'étranger, sur la question de l'accueil des réfugiés, on a besoin de l'invention d'une contre-parole dans la question de l'immigration par exemple. Dans cette ligne-là, le livre fondamental c'est Le monolinguisme de l'autre. Il s'agit alors de tirer des implications politiques au-delà de celles que Derrida tire lui-même.

V. G. : Si une contre-parole est, d'une certaine manière, condition de la démocratie, alors démocratie et politique sont deux choses distinctes.

M. C. : Dans le fond, tout ça consiste à ne pas se satisfaire des conditions d'exercice actuel de la démocratie. Tout ça consiste à se dire que la démocratie telle qu'elle est pratiquée aujourd'hui n'est pas la démocratie. C'est la démocratie et pas la démocratie, en même temps, il ne faut pas appeler autoritaire ce qui ne l'est pas. Mais c'est vraiment crucial de disjoindre l'idée qu'on peut se faire de la démocratie comme promesse de son exercice concret actuel. C'est cette disjonction qui est produite par la déconstruction, dont le geste consiste à ne pas enfermer la démocratie dans le cadre dans lequel elle est déjà instituée et constituée.

V. G. : Jacques Rancière dit que la démocratie n'est pas un régime gouvernemental, mais par contre il transforme la politique en démocratie.

M. C. : C'est très embêtant car son schéma ne permet pas de distinguer la démocratie des autres régimes politiques. Dans un livre que j'ai écrit il y a quelques années, Elections, il y a un long chapitre où je discute avec Rancière, Badiou et Stiegler. J'y consacrerai mon séminaire l'année prochaine, qui portera sur l'articulation entre éthique et politique comme enjeu de la démocratie. 
J. A. : Par rapport aux immigrants - ce qui nous a beaucoup intéressé aujourd'hui -revenons sur la position actuelle de l'Allemagne. Croyez-vous que l'Allemagne se soit transformée en une sorte d'exemple pour l'Europe quant à l'ouverture dont elle a fait preuve face à l'arrivée des réfugiés?

M. C. : Ce qui est certain c'est que l'Allemagne s'est retrouvée très seule pour faire face à cet afflux considérable des réfugiés : il est vrai que pour des raisons liées à la natalité, l'Allemagne a besoin aussi de cette population d'immigrés plus que d'autres pays. Mais elle a aussi sauvé l'honneur de l'Europe, en ouvrant ses portes, là où tant d'autres pays les ont fermées. Maintenant, il faut avouer que cette crise a fait voler en éclats le rêve européen. Ce qui est dramatique, en outre, et consternant, c'est la réaction des populations européennes qui se traduit par la montée de la xénophobie, du nationalisme, des replis identitaires. Voilà qui fragilise considérablement l'Europe, ce qu'elle peut représenter, ce qu'elle peut symboliser! Probablement que si dès le début on n'avait pas laissé l'Allemagne toute seule, la réaction des populations européennes serait en partie différente.

Fait à Paris, le 10 novembre 2015

Recibido : $14 / 10 / 2016$

Aceptado : 13/03/2017

\section{@(9)}

ENDOXA está bajo una licencia de Creative Commons Reconocimiento-NoComercial-SinObraDerivada 4.0 Internacional 